\title{
Based on Short Motion Paths and Artificial Intelligence Method for Chinese Chess Game
}

\author{
Chien-Ming Hung \\ Department of Power Mechanical Engineering, Army Academy R. O. C., Jhongli, City, Taoyuan 32092, Taiwan \\ Jr-Hung Guo \\ Department of Electrical Engineering, National Yunlin University of Science \& Technology, 123 Sec. 3, University Road \\ Douliou, Yunlin 64002, Taiwan \\ Kuo-Lan Su* \\ Department of Electrical Engineering, National Yunlin University of Science \& Technology, 123 Sec. 3, University Road \\ Douliou, Yunlin 64002, Taiwan \\ E-mail: tpemail77@yahoo.com.tw,g9710801@yuntech.edu.tw,sukl@yuntech.edu.tw
}

\begin{abstract}
The article develops the decision rules to win each set of the Chinese chess game using evaluation algorithm and artificial intelligence method, and uses the mobile robot to be instead of the chess, and presents the movement scenarios using the shortest motion paths for mobile robots. Player can play the Chinese chess game according to the game rules with the supervised computer. The supervised computer decides the optimal motion path to win the set using artificial intelligence method, and controls mobile robots according to the programmed motion paths of the assigned chesses moving on the platform via wire less RF interface. We uses enhance $\mathrm{A}^{*}$ searching algorithm to solve the shortest path problem of the assigned chess, and solve the collision problems of the motion paths for two mobile robots moving on the platform simultaneously. We implement a famous set to be called "wild horses run in farm" using the proposed method. First we use simulation method to display the motion paths of the assigned chesses for the player and the supervised computer. Then the supervised computer implements the simulation results on the chessboard platform using mobile robots. Mobile robots move on the chessboard platform according to the programmed motion paths and is guided to move on the centre line of the corridor, and avoid the obstacles (chesses), and detect the cross point of the platform using three reflective IR modules.
\end{abstract}

Keywords: Evaluation algorithm, artificial intelligence method, wireless RF interface, enhance $\mathrm{A}^{*}$ searching algorithm

\section{Introduction}

Chinese chess game is one of the most popular games, and is similar to Western chess to be a two-player game with a complexity level, and is classified red side and black side. In the recent, the Chinese chess game has gradually attracted many researchers' attention, and many evolutionary algorithms to be proposed. Darwen et al. proposed the co-evolutionary algorithm to solve problems where an object measure to guide the search process is extremely difficult to device [1].
In the paper, we use the multi-robot system to present the scenario of the Chinese chess game, and use enhance $A^{*}$ searching algorithm to program the shortest path for mobile robots (chesses) moving to the target points. Player moves the chess to the assigned location or takes the chess of the supervised computer. In other words, the supervised computer can move the chess to take chess of the player, too. Then there are two chesses (robots) moving in the platform simultaneously. The assigned two robots may collide on the programmed motion paths. The proposed algorithm can solve the 
collision condition of two mobile robots and improve the shortest motion path using enhance $\mathrm{A}^{*}$ searching algorithm $[2,3]$

In some condition, the mobile robot must program the shortest path and avoid the other chess moving to the next position. $\mathrm{A}^{*}$ heuristic function is introduced to improve local searching ability to estimate the forgotten value [4]. Flavio et al. presented a multi-robot exploration algorithm that aims at reducing the exploration time to minimize the overall traverse distance of the robots by coordinating the movement of the robots performing the exploration [5].

\section{System Architecture}

The system architecture of the Chinese chess game system is shown in Fig. 1. The system contains a supervised computer, some wireless RF modules, a grid based platform, thirty-two mobile robots and some wireless modules. The game is classified red side (User) and black side (The supervised computer), and belongs to two players. Each side includes sixteen chesses. Player moves chess using the mouse on the user interface, or takes chess of the other side. The chess game will be ending until the king of each side to be taken by another side.

We want to increase the entertainment function using mobile robots to present the movement scenarios of the chesses. The supervised computer is a player to compete with the user, and programs the shortest motion path using enhance $A^{*}$ searching algorithm and transmits the ID code and motion command to the assigned mobile robots. The assigned mobile robots receive the self-ID code and the target positions via wireless RF interface, and move to the assigned positions to avoid the collision paths according to the programmed motion paths.

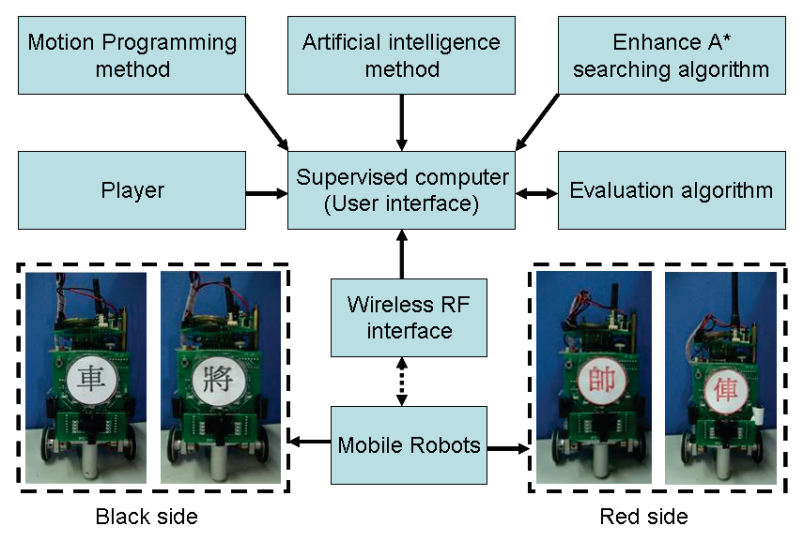

Fig. 1. System architecture
In the collision problem, player moves chess to take chess of the other side. The user interface programs two motion paths for the assigned chesses. Two mobile robots avoid collision points and paths according to the programmed motion paths moving on the platform. Players can set game time of each set and limit moving time of each step for red side and black side. Players don't obey the game rules to move chess using the mouse. The supervised computer can't permit and display the movement status on the user interface.

\section{Motion Planning}

The mobile robot has the shape of cylinder, and it's equipped with a microchip (STC12C5A60S2) as the controller, two DC servomotors and two driver devices, some sensor circuits (contain compass circuit), a voice module, three $\mathrm{Li}$ batteries, a wireless RF interface $(2.4 \mathrm{GHz})$ and three reflect IR sensor modules (One module contains two reflective IR sensors). Meanwhile, the mobile robot has four wheels to provide the capability of autonomous mobility. The structure of the mobile robot is shown in Fig. 1.

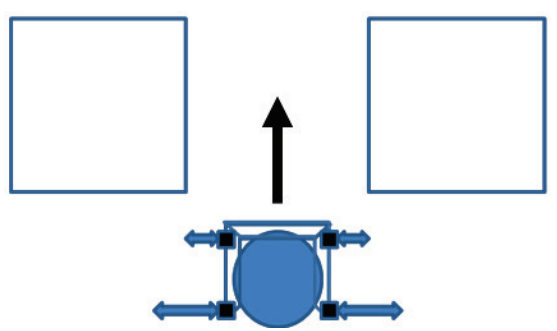

(a)

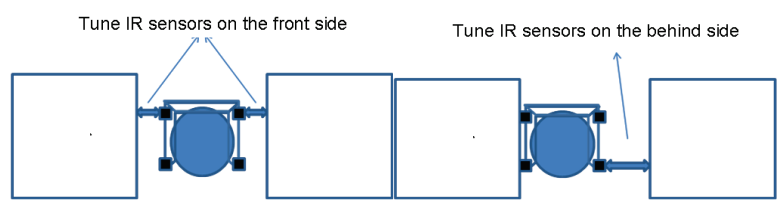

(b)

(c)

Fig. 2. The detection range for reflective IR modules

The mobile robot uses three reflective IR modules to detect the wall of each grid and obstacles. Two reflective IR sensors are fixed on the both sides of the mobile robot. The detection distance of the front side is shorter than the behind side shown in Fig. 2(a). In the both side's modules, two reflective IR sensors of the front side can detect distance to control the mobile robot moving in the centre line of the corridor shown in Fig. 2(b). Two reflective IR sensors can detect the maximum 
distance to be equal to the width of the corridor minuses the width of the mobile robot to be fixed on the behind side of the module shown in Fig. 2(c). The other reflective IR module is fixed on the front side, and detects the obstacle or the mobile robot on the motion path.

$\mathrm{A}^{*}$ searching algorithm solves the path planning problem of multiple nodes travel system. The formula of $\mathrm{A}^{*}$ searching algorithm is following

$$
f(n)=g(n)+h(n)
$$

The core part of an intelligent searching algorithm is the definition of a proper heuristic function $f(n) . g(n)$ is the exact cost at sample time $n$ from start point to the next point. $h(n)$ is the minimum cost. In this study, $n$ is reschedules as $n^{\prime}$ to generate an approximate minimum cost schedule. The equation (1) can be rewritten as follows:

$$
f(n)=g(n)+h\left(n^{\prime}\right)
$$

The A* searching algorithm can program local minimum motion path. We improve $\mathrm{A}^{*}$ searching algorithm that is called enhance $A^{*}$ searching algorithm that can search the shortest motion path for mobile robots [2]. In the Chinese chess game, the chesses of red side must face to the black side. In the same way, the chesses of black side must face to red side, too. The enhance $A^{*}$ searching algorithm can delete the turning numbers to decrease the total motion distance, and select all cross points from the programmed motion path to cut down redundant motion path in the rectangle region.

\section{Experimental Result}

We make an example to explain how to implement in Chinese chess game, and use evaluation algorithm and artificial intelligence method to decide the moving chess with the highest evaluation score, and use enhance $A^{*}$ searching algorithm to program the shortest motion paths of the assigned chesses from the start positions to the target positions. There is a famous set to be called "wild horse run in farm" shown in Fig. 3.

There are eight chesses (one king, two elephants, two rooks, one horse, two pawns) in red side and nine chesses (one king, two advisors, two elephants, one rook, and three pawns) in the black side for the famous set. Movement process of the set is belonging to the player and the supervised computer shown in Table. 1 to be classified four steps. In the first step, the player moves red rook form $(8,5)$ to $(8,1)$. The motion path of the chess "red rook" is programmed using enhance $A^{*}$ searching algorithm. The movement scenario of the assigned chess is shown in left-up side of Fig. 4(a) on the user interface. The movement status of the mobile robot is instead of the assigned chess shown in Fig. 4(a). Then the computer moves the black advisor to protect the king from $(5,2)$ to $(6,1)$. The movement scenario of the assigned chess is shown in Fig. 4(b).

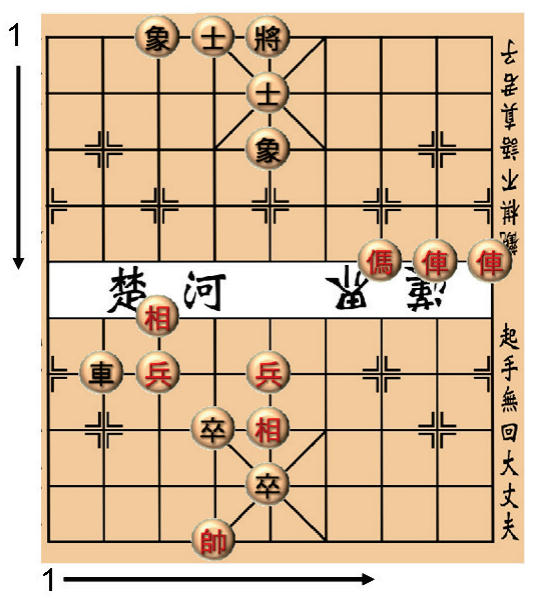

Fig. 3 A famous set "Wild horses run in farm"

Next the player moves the red horse to take the black king from the position $(7,5)$ to the position $(6,3)$, and the computer moves the black king from the position $(5,1)$ to $(5,2)$ in the second step. The movement process of the grid based platform is shown in Fig. 4(c) and (d). Each step of the computer must calculate the score of the moveable chesses shown in Table 1. Then the computer decides the optimal motion path of the selected chess. In the third step, the player moves the red rook from $(8,1)$ to $(8,2)$, and the computer moves the black elephant from $(3,1)$ to $(1,3)$ shown in Fig. 4(e) and (f). Then the black king will be taken by the red rook in the fourth step of the red side, and the game will be ending. The movement scenarios of two chesses (two mobile robots) are shown in Fig. 4(g). The motion paths of two mobile robots have collision problem in the case. The supervised computer must re-program the new motion paths to avoid the collision path. The black king is taken by the red rook, and moves to the assigned position. The set will be ending. The final arrangement positions of the remainder chesses shown in Fig. 4(h). 
Table 1 Movement process of the assigned set

\begin{tabular}{|c|c|c|c|}
\hline & \multirow{2}{*}{ Player (red side) } & \multicolumn{2}{|c|}{$\begin{array}{c}\text { Computer (black side, using } \\
\text { evaluation algorithm) }\end{array}$} \\
\cline { 3 - 4 } First step & $\begin{array}{c}\text { Rook } \\
(8,5) \rightarrow(8,1)\end{array}$ & $\begin{array}{c}\text { Advisor }(5,2) \\
\rightarrow(6,1)\end{array}$ & -295 \\
\hline $\begin{array}{c}\text { Second } \\
\text { step }\end{array}$ & $\begin{array}{c}\text { Horse }(7,5) \\
\rightarrow(6,3)\end{array}$ & King $(5,1) \rightarrow(5,2)$ & -18888 \\
\hline $\begin{array}{c}\text { Third } \\
\text { step }\end{array}$ & $\begin{array}{c}\text { Rook }(8,1) \\
\rightarrow(8,2)\end{array}$ & $\begin{array}{c}\text { Elephant }(3,1) \\
\rightarrow(1,3)\end{array}$ & -19991 \\
\hline $\begin{array}{c}\text { Fourth } \\
\text { step }\end{array}$ & $\begin{array}{c}\text { Rook (8,2) } \\
\rightarrow(5,2)\end{array}$ & & \\
\hline Result & Winner & & \\
\hline
\end{tabular}

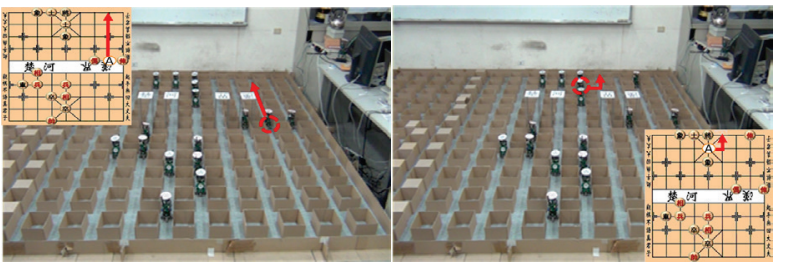

(a)The first step (player)

(b)The first step(computer)

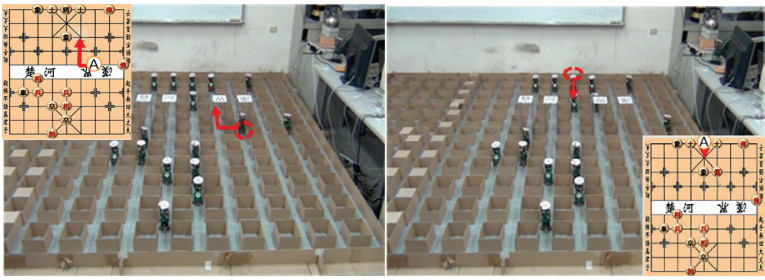

(c)The second step (player) (d)The second step (computer)

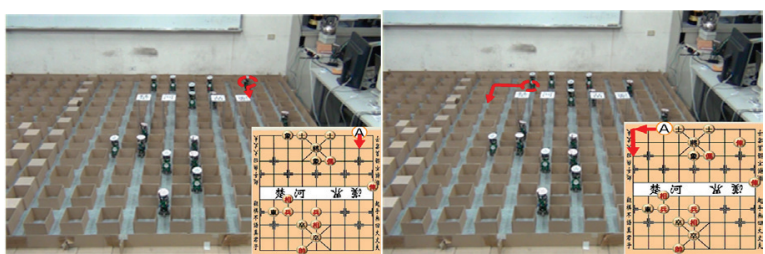

(e)The third step (player)

(f)The third step (computer)

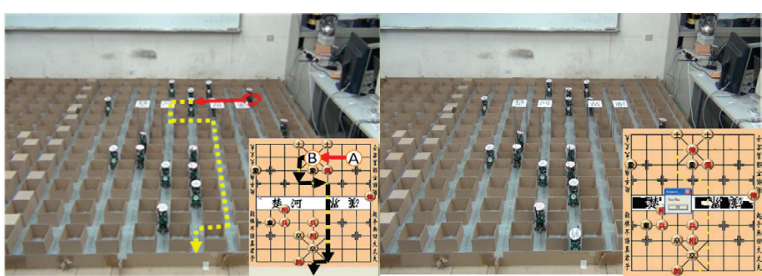

(i) The final step (player)

(j) The final arrangement

Fig. 4. Moving process for the set

\section{Conclusion}

The Chinese chess game system contained a supervised computer with the user interface, some wireless RF modules, a chessboard platform and thirty-two mobile robots. The supervised computer can select moveable method of the assigned chess using evaluation algorithm and artificial intelligence method. We programmed the shortest motion paths of assigned chesses using enhance $A^{*}$ searching algorithm to obey the rules of Chinese chess game. The proposed methods can program two motion paths for two chesses, and solve the collision problem for multiple mobile robots moving on the chessboard platform simultaneously. We implemented a famous set to be called "wild horses run in farm" using the proposed method. The paper used the set to implement the evaluation algorithm, artificial intelligence method and enhance $A^{*}$ searching algorithm. The proposed methods were not only used in the Chinese chess game, but also applied in various game, and entertainment field, and manufacture process and production management. Further the moving process of mobile robots can be used in the simultaneous war using the proposed algorithms.

\section{Acknowledgements}

This work was supported by the Ministry of Science and Technology in Taiwan, (MOST 105-2221-E-224-022).

\section{References}

1. Darwen P. and Yao X., Coevolution in Iterated Prisoner's Dilemma with Intermediate Levels of Cooperation: Application to Missile Defense, International Journal of Computational Intelligence Applications, 2(1) (2002), pp.83-107.

2. Su K. L., Li B. Y. and Chung C. Y., Enhance A* Searching Algorithm Applying in Multiple Robot System, Applied Mechanics and Materials, Vol.479-480 (2014), pp.773-777.

3. Su K. L., Su S. T., Hsia K. H. and Li B. Y., Implementation of the Chinese Chess Game with the Computer Using Artificial Intelligence, International Journal of Research and Surverys, ICICExpress Letters, Part B: Applications, 7(6) (2016), pp.1353-1358.

4. Fu T. and Yin H., Designing a hybrid position evaluation function for Chinese-chess computer game, International Conference on Software Engineering and Service Science, (2013), pp.75-78.

5. Saber Y. and Senjyu T., Memory-bounded ant colony optimization with dynamic programming and $\mathrm{A}^{*}$ local search for generator planning, IEEE Trans. on Power System, 22(4) (2007), pp.1965-1973.

6. Su K. L., Li B. Y., Guo J. H. and Hsia K. H., Implementation of The Chess Game Artificial Intelligent Using Mobile Robot, International Conference on Soft Computing and Intelligent System, (2014), pp.169-174. 